\title{
Representaciones maternas y transmisión de los factores de riesgo y protección en hijos de madres drogodependientes
}

\author{
Fava Vizziello, G. ${ }^{(1)}$; Simonelli, A. ${ }^{(2)}$; Petenà, I. ${ }^{(3)}$
}

(1) Profesora de Psicopatología Evolutiva. Departamento de Psicología del Desarrollo y de la Socialización. Universidad de Padua. (2) Doctora de Investigación. Departamento de Psicología del Desarrollo y de la Socialización. Universidad de Padua. (3) Psicóloga especialista en Psicología Clínica. Universidad de Pauda.

\section{RESUMEN}

La investigación consiste en la valoración de los Modelos Operativos Internos del vínculo entre las madres drogodependientes y sus hijos, así como de la transmisión de dicho modelo del vínculo a través de varias generaciones. El estudio se ha centrado en 28 madres drogodependientes residentes en comunidades terapéuticas; de este grupo se extrajo posteriormente un subgrupo de 16 díadas de madres e hijos. Para la valoración del vínculo en las madres se ha empleado la Adult Attachment Interview de George, kaplan y Main (1985) y para los hijos, la Strange Situation (Ainsworth, Blehar, Waters, \& Wall, 1978). Se han obtenido los siguientes resultados: en las madres drogodependientes se advierte un predominio de vínculos poco seguros (Rechazo, 39,3\% o Complicados, 53,6\%), diverso del que aparece en madres que no lo son; en sus hijos se ha revelado una presencia de vínculos poco seguros (Evitativos, $24 \%$ o Ambivalentes, $31,2 \%$ ) con un importante porcentaje de vínculo desorganizado (25\%), un resultado que, sin embargo, no se diferencia de la distribución del vínculo en la población general infantil; en lo que respecta a la correspondencia entre los modelos de vínculo de la madre y los del hijo, el examen de tres categorías de vínculo no ha revelado asociaciones estadísticamente significativas y sólo en los resultados del análisis de la dicotomía seguridad vs. inseguridad han aparecido resultados que podrían considerarse significativos. Estos resultados parecen demostrar, pues, que los hijos tienen una capacidad de adaptación mejor que la de las madres y pueden considerarse puntos de partida para reflexiones que contemplen tanto los factores evolutivos y de madurez específicos del niño como la posibilidad de que la comunidad en la que residen madre e hijo desempeñe un rol de protección.

Palabras clave: factores de riesgo, madres adictas, hijos de adictos, dinámica familiar, comunidades terapéuticas, teoría del vínculo.

\section{SUMMARY}

This study is interested in the evaluation of Internal Working Models in pairs of addicted women and their children living in residential therapeutic communities and in a possible transmission of attachment patterns through the generations. 28 women were considered: from this sample was extracted a sub-group of 16 pairs mothers-children. Attachment quality was studied through the application of the Adult Attachment Interview by George, Kaplan and Main (1985) in mothers and of the Strange Situation (Ainsworth, Blehar, Waters, \& Wall, 1978) in their children. The results show the existence of specific features about the distribution of attachment patterns in addicted women compared to the non addicted ones: addicted women display in fact insecure attachment organizations (39,3\% Dismissing, and 53,6\% Entangled-Preoccupied). Another finding is connected to the presence of insecure attachment patterns in their children (24\% Avoiding, 31,2\% Ambivalent and 25\% Disorganized); this result, however, doesn't contrast with attachment distributions in normal children population. As to the agreement between mother's attachment models and children's ones, there are no main data but only a tendency to statistic significance when we consider secure vs. insecure attachment patterns.

These findings underline how addicted women's children can organize more adaptive strategies than their mothers. A particular interest is reserved to this aspect, which could be linked to different aspects: on one hand to child's developmental factors, on the other to the protective role played by residential therapeutic communities which can offer alternative attachment figures and an supportive environment.

Key words: addict mothers, risk factors, addict children, family dynamic, therapeutic communities, attachment theory. 
E esquema teórico del vínculo se viene centrando cada vez más en el estudio del desarrollo del niño en el contexto en el que tiene lugar la crianza. Concretamente, algunas de las contribuciones más interesantes sobre este tema proceden de investigaciones llevadas a cabo en situaciones de riesgo progresivo en las que existe una interconexión entre factores personales, de relación y sociales que no respalda la adaptación del individuo al ambiente que lo rodea. En esta misma línea, Cicchetti y Rizley (1981) distinguen dos clases de factores de riesgo para el desarrollo: factores internos del niño, como anomalías físicas, comportamentales y trastornos psicológicos que dificultan la atención que le dedican los padres y factores externos al niño, o lo que es lo mismo, relacionados con el contexto en el que crece, como la historia personal del progenitor, sus características psicológicas o elementos ecológicos relativos tanto a su entorno personal como al de la familia completa.

En el estudio del grupo de niños que se trata en este trabajo, el de hijos de progenitores (especialmente madres) drogodependientes, deben tenerse en cuenta los dos. En el análisis de su desarrollo afectivorelacional la interconexión entre riesgos endógenos y exógenos es un instrumento fundamental.

\section{FACTORES ENDÓGENOS DE RIESGO}

Muchas de las investigaciones que se llevan a cabo sobre hijos de madres drogodependientes se ocupan de los efectos de la exposición del feto a sustancias psicotrópicas. Entre los efectos perinatales de las sustancias psicoactivas en recién nacidos expuestos al consumo de cocaína y heroína durante el periodo de gestación, los autores destacan rotura de placenta y nacimiento prematuro; bajo peso en el nacimiento y niveles de APGAR inferiores a los normales; menor circunferencia craneal; incidencia de infartos cerebrales perinatales; malformaciones congénitas y trastornos neurocomportamentales, (Lutinger, Graham, Einarson, \& Karen, 1991; Mayes, Granger, Bornstein, \& Zuckerman, 1992; Zuckerman \& Bresnahan, 1991; Zuckerman et al., 1989). Además, el feto puede establecer una dependencia de las drogas que toma la madre, con el riesgo de sufrir inmediatamente después del parto, a causa de la brusca interrupción del suministro de droga, verdaderas crisis de abstinencia, que varían según el tipo de sustancia de que se trate y de la modalidad en que se suministrase (Finnegan, 1986). Debe subrayarse, finalmente, el hecho de que muchos de estos niños nacen en condiciones de seropositividad al VIH, que en la mayor parte de los casos remite tras los primeros meses de vida, pero que en otros casos termina infectándole. Estos datos también deben aplicarse en otros tipos de patologías infeccio- sas como hepatitis, sífilis, toxoplasmosis o citomegalovirus (Zacchello, \& Giaquinto, 1997).

En edades posteriores, algunos estudios refieren dificultades de aprendizaje, menor capacidad de atención y una mayor agresividad en edad pre escolar y escolar (Cavazzuti, Frigieri, \& Finelli, 1987; Fundaro \& Salvataggio, 1987; Oloffson \& Bueckley, 1983; Sanderegger \& Zimmermann, 1978; Wilson, McCreary, kean, \& Baxter, 1979), aunque no se advierten diferencias en los niveles de Cl (Azuma \& Chasnoff, 1993) ni alteraciones de las funciones intelectivas diversas a las de los niños de los grupos de control. En este sentido, Lester y Tronick (1994) proponen que el análisis de los efectos de la exposición prenatal a las drogas considere las dificultades funcionales en el ámbito de las $4 \mathrm{~A}$ de la infancia (Atención, Arousal [Estímulo], Afectividad y Acción). La vulnerabilidad neurocomportamental que caracteriza a los niños expuestos a los efectos de las drogas en el útero se inserta, pues, en un contexto más amplio en el que también tienen cabida los factores relacionales y ambientales que influyen en el desarrollo del pequeño en los primeros momentos que siguen al nacimiento.

\section{FACTORES AMBIENTALES: LA APORTACIÓN DEL VÍNCULO}

Junto a los numerosos trabajos citados más arriba también es necesario considerar diversos efectos postnatales que contribuyen a determinar los logros evolutivos de los hijos de drogodependientes. Entre ellos destacan los que podríamos definir como factores ambientales próximos, o sea, las modalidades de atención y protección que el niño obtiene de su ambiente primario, es decir, de la madre (o de los adultos encargados de cuidarlo). En los últimos años uno de los instrumentos clave para la lectura teórica y metodológica en el estudio del desarrollo afectivo-relacional en los primeros años de vida en poblaciones de riesgo es el cuadro del vínculo, y a su alrededor se han desarrollado muchas de las investigaciones y reflexiones clínicas sobre estos temas.

Concretamente, según este modelo teórico, en el primer año de vida el principal logro evolutivo del niño es la construcción de la seguridad (Sroufe \& Waters, 1977), además de uno de los factores que lo protegen en el proceso de adaptación a su ambiente en la medida en que haya vivido experiencias relacionales de apoyo y respuesta ante sus necesidades y demandas (Ainsworth, Blehar, Waters, \& Wall, 1978).

En este sentido, existen varias investigaciones que han intentado dilucidar cuál es el papel jugado por el progenitor y por el pequeño en la caracterización de la cualidad del vínculo entre ambos, aún cuando no esté clara la aportación de cada uno. Una de las aportaciones más interesantes es el trabajo de meta análisis de 
van IJzendoorn, Goldberg, Kroonenberg y Frenkel (1992) sobre el peso relativo de las problemáticas del niño o del progenitor en el desarrollo del vínculo durante el primer año de vida, evaluado a través de la Strange Situation de Ainsworth, et al. (1978). Estos autores han encontrado una menor presencia de vínculo Seguro (B) y un incremento de las proporciones de vínculo Desorganizado/Desorientado (D) en muestras compuestas de díadas madre-hijo de riesgo, que eran diferentes a las distribuciones que surgían en el ámbito de la población general. Además, al determinar la cualidad del vínculo se reveló que las problemáticas y las dificultades maternas tenían una influencia mucho mayor que los factores de riesgo internos del niño. De hecho, en los grupos de niños con madres que maltrataban (Carlson, Cicchetti, Barnett, \& Braunwald, 1989; Schneider-Rosen, Braunwald, Carlson \& Cicchetti, 1985), que tenían trastornos mentales o problemas de drogodependencia (Rodning, Beckwith, \& Howard, 1989) las distribuciones del vínculo eran muy diferentes a las que aparecían en todas las muestras normalizadas elegidas como referencia, superiores a las que se evidenciaban cuando las problemáticas eran de los niños. Por el contrario, los problemas del niño no parecen comprometer la construcción de un vínculo seguro con la madre (van IJzendoorn et al., 1992).

A su vez, las investigaciones con grupos de progenitores con patologías psiquiátricas, problemas de comportamiento o actitudes de maltrato hacia los hijos también parecen seguir la misma dirección, evidenciando altos porcentajes de vínculo poco seguro, concretamente, de vínculo Complicado (E) y No Resuelto en relación con un Duelo o un Trauma (U), que pueden derivarse de la dificultad de estas personas para reelaborar experiencias vitales incorrectas y de inseguridad experimentadas durante la infancia respecto a sus propias figuras de vínculo (van IJzendoorn \& Bakermans-Kranenburg, 1996). Por ello en los grupos de progenitores que pertenecen a poblaciones clínicas la ausencia de seguridad parece ser un factor de riesgo potencial para el hijo a causa del proceso de transmisión del vínculo, según el cual el mundo representativo de la madre asume un rol fundamental en la construcción conjunta del vínculo con el pequeño (Benoit \& Parker, 1994; Fonagy, Steele \& Steele, 1991; Ward \& Carlson, 1995; van IJzendoorn \& BakermansKranenburg, 1997; Zeanah, 1992).

\section{LA INVESTIGACIÓN}

En base a lo que ha quedado expuesto, presentamos a continuación un trabajo de investigación realiza- do en dos comunidades para madres drogodependientes y sus hijos elegidas entre las diversas que existen en Italia'. Estas estructuras se comprometen a ocuparse de la residencia de la díada madre-hijo (algunas veces también del padre), brindándoles también un recorrido terapéutico y educativo/rehabilitativo articulado, que se desarrolla durante un periodo de los dos años aproximadamente que dura la estancia (modificable según las distintas necesidades). Concretamente, en las comunidades estudiadas, el modelo de intervención psicológica propuesto consiste en una combinación de psicoterapia individual y terapias de grupo, en los dos casos guiadas por psicoterapeutas con formación psicodinámica y/o psicoanalítica.

En el territorio italiano existen actualmente numerosas comunidades para las madres drogodependientes y sus hijos, con notables diferencias entre sí, en virtud de la compleja normativa que articula su organización y definición (puestos disponibles, estructuras, cuotas mensuales, etc.), que gozan de autonomía regional. En general se trata de casas situadas en contextos periféricos de ciudades o en pueblos con amplios espacios internos y/o externos disponibles para las actividades de los niños: cada una acoge hasta 10/12 parejas con una edad para los niños que oscila entre los pocos meses (algunas veces, sin embargo, también se acogen madres embarazadas) a los 6-7 años.

La investigación se encuadra en el contexto de los estudios sobre el vínculo, en un intento de aislar algunos indicadores clínicos relacionados con el desarrollo afectivo-relacional del niño durante los dos primeros años de vida y, paralelamente, con la cualidad de la relación que mantiene con la madre. Uno de los objetivos del estudio es analizar la influencia del mundo representativo de la madre, vinculado a su historia relacional, en el origen de los comportamientos que tienen que ver con su capacidad de responder a las necesidades de su hijo y, por tanto, en la construcción de la relación primaria con él. Desde ese punto de vista, la teoría del vínculo aparece como un paradigma muy interesante para la conceptualización de la transmisión, mediante un mecanismo de interacción, de la cualidad de las representaciones de las experiencias relacionales primarias ligadas a la capacidad de respuesta y protección de los adultos más relevantes. Estas propuestas son especialmente eficaces para la lectura de situaciones clínicas, entre las que se sitúan las de las madres drogodependientes.

En este sentido, el trabajo ha empezado analizando las diferencias y las similitudes que existen en la distribución de las características de las representacio-

\footnotetext{
"Damos las gracias a las comunidades "Villa Renata" del Lido de Venecia y "San Francesco" de Monselice por habernos facilitado tiempo y espacios y por su colaboración en el trabajo de reflexión clínica y de investigación que hemos llevado a cabo en ellas.
} 
nes mentales de la experiencias y de las figuras de vínculo (construidas a partir de las vivencias personales) de un grupo de madres drogodependientes, comparándolas con las de un grupo de madres de la población general muy similar al primero en las edades de los sujetos y de sus hijos. Concretamente, en el grupo clínico se espera encontrar proporciones de vínculo Seguro (F) significativamente inferiores a las del grupo de control, además de un modelo predominante que caracterice las peculiaridades de estos sujetos. Finalmente, teniendo en cuenta las biografías personales, extremadamente difíciles, de muchas de estas mujeres, se prevé la presencia de elevadas proporciones de vínculo No Resuelto en relación con un Duelo o con un Trauma (U).

El segundo objetivo ha consistido en la valoración del posible factor de riesgo de las representaciones maternas de vínculo inseguro o no resuelto en situaciones luctuosas o traumáticas para el desarrollo afectivo-relacional del niño; la influencia de esas representaciones sobre el desarrollo infantil, de hecho, se manifiesta a través de la sensibilidad con la que el progenitor acoge las señales del hijo y responde a ellas de modo adecuado, determinando sus comportamientos de atención y protección hacia él (Ainsworth, et al. 1978; van IJzendoorn, 1995). Para esto se ha intentado examinar la transmisión del vínculo entre madre e hijo en el interior de estas díadas, con el propósito de comprender mejor algunos de los mecanismos que determinan la influencia de la cualidad de las representaciones maternas de vínculo sobre la cualidad del vínculo del hijo, tanto en lo que respecta a la proporción de seguridad como en lo referente a los otros modelos de vínculo.

En resumen, el trabajo intenta determinar posibles elementos de protección que contribuyen a modificar, en términos de adaptación, el posible carácter lineal de la transmisión de la inseguridad entre madre e hijo: en este caso, el trabajo de acogida y de intervención desarrollado por la comunidad podría asumir un rol relevante como factor de mediación respecto a la cualidad del vínculo desarrollado por el niño en su relación con la madre.

\section{METODOLOGÍA DE LA INVESTIGACIÓN}

\section{Los participantes.}

Para verificar las hipótesis enunciadas se ha llevado a cabo una investigación sobre un grupo de 28 madres drogodependientes que llevasen residiendo un mínimo de seis meses en las comunidades terapéuticas para madres e hijos que hemos descrito anteriormente.

Las 28 madres elegidas tienen una edad comprendida entre los 20 y los 36 años $(x=28.9)$, un nivel de escolarización más bien bajo (26 sujetos poseen un diploma de Escuela Media Inferior (en España equivaldría a la enseñanza primaria] o profesional). En relación con el estado civil, 9 sujetos son casados y 19 son solteros. $Y$ en lo que respecta a las condiciones en las que ha discurrido el crecimiento de estas mujeres en las familias de origen, 14 de ellas han visto y/o sufrido maltratos y 4 han sido víctimas de abusos sexuales (también externos a la propia familia); en 12 de los casos considerados se asiste a la presencia de síntomas psicopatológicos en al menos uno de los miembros de la familia de origen (alcoholismo, generalmente de los padres, y trastornos psiquiátricos de la madre).

Del grupo de madres se ha extraído un subgrupo de 16 díadas madre-hijo, en base a una edad del pequeño comprendida entre los 13 y los 24 meses (edad media $x=20$ meses), de modo que pudiese llevarse a cabo una aplicación de la Strange Situation para la valoración del vínculo en la primera infancia. Las madres tienen una edad comprendida entre los 20 y los 25 años (edad media $x=29$ años). En relación con el estado civil, el 38\% ( $N=6)$ es de casadas, el 19\% $(N=3)$ es de separadas o divorciadas, el 31\% (N=5) es de solteras y el $12 \%(\mathrm{~N}=2)$ convivía con la pareja antes de ingresar en la comunidad. La sustancia de abuso principal es la heroína en un 69\% (11 sujetos), el alcohol en 3 sujetos, la heroína y el alcohol en 1 sujeto y cocaína en 1 sujeto. Además, 12 de ellas (casi un 75\%) tienen una pareja con un historial pasado o vigente de drogodependencia. Los 16 niños son un 50\% de sexo masculino y un $50 \%$ de sexo femenino.

Además de los casos de hospitalización precoz provocados por la condición de drogodependencia de las madres, se observa que 3 niños han mostrado en el momento de nacer síndrome de abstinencia neonatal, y 1 niño síndrome de alcoholismo fetal (SAF), sin consecuencias sobre el desarrollo psico-físico normal. Antes de que las madres ingresasen en la comunidad, había 3 niños al cuidado de la abuela materna, 3 al de instituciones de acogida, el 56\% (N=9) a las madres naturales y sólo 1 estaba en situación de preadopción.

\section{Instrumentos para la recogida de datos.}

Los instrumentos utilizados para verificar las hipótesis han sido los siguientes:

-Todas las mujeres participantes han sido sometidas a la Adult Attachment Interview (AAI, George, Kaplan, \& Main, 1985).

-Los niños han sido sometidos a la Strange Situation (SSP= de Ainsworth et al. (1978) para evaluar el tipo de vínculo en las relaciones con la madre el estilo de vínculo en las relaciones con la madre. La SSP es un procedimiento de laboratorio que consta de ocho episodios de alrededor de 
3 minutos de duración, cuya secuencia se dirige a someter al niño a niveles de estrés crecientes con el fin de hacer surgir sus comportamientos de vínculo hacia la figura de referencia.

\section{Métodos de clasificación de los datos.}

Las AAl se han transcrito palabra por palabra, según las normas establecidas y codificadas por dos árbitros independientes con el método propuesto por los autores (Main \& Goldwyn, 1994). De este modo ha sido posible asignar una de las siguientes categorías de vínculo: Rechazo (Dismissing, Ds), Seguro (FreeAutonomus, F), Complicado-Preocupado (EntengledPreoccupied, E), Vínculo no Resuelto respecto a un Duelo o a un Trauma (Sin resolver, U)².
Las Strange Situation se codifican a través de dos árbitros independientes que identifican, en primer lugar, uno de los modelos principales de vínculo, Evitativo (A), Seguro (B), o Ambivalente (C), según el procedimiento descrito por Ainsworth et al. (1978) y, a continuación, asignan la categoría de Desorganizado/Desorientado (D), según el esquema elaborado por Main \& Salomon $(1986,1990)^{3}$.

\section{Métodos de análisis de los datos.}

Los datos han sido analizados con diversos procedimientos:

-A nivel cualitativo, se han estudiado frecuencias y porcentajes, con el fin de obtener elementos descriptivos generales de las distribuciones de las categorías del vínculo.

\footnotetext{
${ }^{2}$ Main y Goldwyn (1994) distingue las siguientes categorías de vínculo:

Rechazo (Dismissing:Ds): se atribuye a los individuos que intentan limitar activamente la influencia de las experiencias y de las relaciones de vínculo en la vida real. Normalmente organizan el pensamiento de modo que puedan tener el vínculo relativamente desactivado a través de la idealización de las experiencias y de las figuras de vínculo, de la supresión del recuerdo o de una desvalorización displicente. La experiencia real de estos sujetos en la infancia ha estado frecuentemente marcada por carencias afectivas por parte de las figuras de vínculo bien por distancia afectiva o bien por comportamientos de rechazo.
}

Vínculo Seguro (Free-Autonomous; F): durante la entrevista, los sujetos seguros se muestran libres para explorar lo que sienten y lo que piensan en relación con las áreas investigadas, aportando una visión coherente de la naturaleza de sus propias experiencias con las figuras significativas de la infancia y, sobre todo, de los efectos de éstas sobre el estado de sus mentes. Todo ello es consecuencia de las experiencias positivas vividas en la infancia o de un activo proceso de reelaboración de experiencias pasadas poco favorables.

Vínculo Complicado-Preocupado (Entengled-Preoccupied; E): se atribuye a los sujetos que manifiestan un cuadro confuso y poco objetivo de la experiencia personal del pasado, del cual puede deducirse un desconcierto, una confusión en el ámbito de las relaciones en el interior de la familia que siguen actuando sobre el estado de la mente. La confusión impide a estos sujetos efectuar un proceso de análisis y reflexión a posteriori objetivo sobre la experiencia del pasado.

Vínculo con Traumas o Duelos No Resueltos (Unresolved; U): se relaciona con la aparición de detalles evidentes de que no se han solucionado determinados episodios de la historia pasada del sujeto que pueden haber representado momentos de grave desorganización del vínculo, como por ejemplo duelos graves, abusos o traumas de otro tipo sufridos en la infancia. Vínculo Inclasificable (Cannot Classify; CC): la entrevista se considera inclasificable cuando el individuo muestra una notable o insólita mezcla de estados mentales que impiden la evidencia de cualquier tipo de estado concreto o de estrategia organizados. No obstante, esta eventualidad aparece raramente en los sujetos pertenecientes a la población normal.

${ }^{3}$ Las categorías de vínculo establecidas por Ainsworth, Blehar, Waters y Wall (1978) para el niño son: Vínculo Seguro (B): el niño muestra angustia en el momento de separación de la madre, un malestar que está más relacionado con la ausencia del progenitor que con el hecho de sentirse solo. El niño busca activamente la cercanía y el contacto con la figura de referencia y también se muestra dispuesto a la interacción con el extraño, evidencia una buena fe en la capacidad de comprensión por parte del adulto y, gracias a ello, está en condiciones de establecer nuevas exploraciones en el ambiente que le rodea. En otras palabras, tiende a manifestar comportamientos de "base segura".

Vínculo Ansioso-Evitativo (A): lo manifiestan los niños que no parecen estresados o ansiosos cuando tienen que separarse del progenitor o que muestran señales de protesta y de angustia que parecen estar más bien ligadas al malestar por encontrarse solos, dado que cesan claramente ante la presencia de cualquier adulto, aunque no sea la madre. En la fase de reencuentro con el progenitor el niño evita el contacto y la interacción con la madre, sin dar muestra de ningún signo de placer cuando se reúne con ella.

Vínculo Ansioso-Ambivalente (C): en el que el niño manifiesta un intenso malestar por la separación, pero también una fuerte ambivalencia y muchos comportamientos de resistencia en el contacto y la interacción con la figura de vínculo alternativa, aunque también tiene momentos de búsqueda de contacto y de cercanía. En estos niños pueden aparecer episodios de desconsuelo después de la separación o de pasividad extrema y ausencia de reacciones. Durante toda la sesión experimental los niños manifiestan comportamientos exploratorios bastante inhibidos.

Vínculo Desorganizado-Desorientado (D): consiste en comportamientos desorganizados y/o desorientados que el niño manifiesta en presencia del progenitor. Estos se manifiestan hasta niveles que llegan a interrumpir el desarrollo de una estrategia de vínculo por parte del niño (Main \& Salomon, 1986, 1990). 
La homogeneidad en la distribución de los grupos comparados entre sí se ha comprobado a través de la estadística del Chi-cuadrado, que distingue el grado de implicación de las distintas variables consideradas, y a través del Modelo de Análisis Múltiple Log-Lineal Saturo ${ }^{4}$ que profundiza en la aparición de la asociación y en la aportación de cada una de las categorías de las variables consideradas a la misma asociación (Cristante, 1992).

Para comprobar la reciprocidad entre las respectivas categorías de las distribuciones de dos grupos de sujetos se ha utilizado el índice K de Cohen.

\section{La conformidad entre los árbitros.}

Todos los datos recopilados han sido valorados por dos árbitros independientes que han alcanzado un nivel de conformidad bastante elevado.

En lo que respecta al grupo de madres drogodependientes, el porcentaje de acuerdo alcanzado entre los árbitros ha sido del 85\% ( $k$ de Cohen $=0.721, p=$ $0.001)$, considerando tres categorías de vínculo -Rechazo (Ds), Seguro (F), Complicado-Preocupado (E)- y del 92\% ( $k=0.89, p=0.001)$ respecto a cuatro categorías -Rechazo (Ds), Seguro (F), Complicado-Preocupado (E), No Resuelto (U)-. El acuerdo en las Strange Situation de los niños ha sido del 85\% ( $k$ de Cohen $=0.72, p=0.001$ ) respecto a tres categorías de víncu- lo -Evitativo (A), Seguro (B), Ambivalente (C)- y del $89 \%(k=0.74, p=0.001)$ respecto a cuatro categorías, incluyendo la categoría Desorganizado/Desorientado (D)

\section{LOS RESULTADOS}

\section{El vínculo en las madres drogodependientes.}

La tabla 1 refleja las distribuciones aparecidas en el grupo estudiado en relación con tres y cuatro categorías de vínculo.

Desde el punto de vista descriptivo se evidencia un predominio de vínculos poco seguros, con una importante presencia de la categoría Complicado-Preocupado (E) que es la que más aparece en el interior del grupo ( $n=15$ igual a 53,6\%), cuando se consideran tres categorías de vínculo. Para verificar estadísticamente los resultados, se ha comparado la distribución del grupo de madres drogodependientes con la de un grupo de madres de la población general italiana (Simonelli, 1998). Esa muestra ha evidenciado una distribución que no sólo concuerda con los enunciados teóricos, sino también con los datos aportados por van IJzendoorn y Bakermans-Kranenburg (1996) en su trabajo de meta análisis sobre aplicaciones del AAl a distintas muestras de sujetos pertenecientes a la población general, procedentes de distintos contextos

\begin{tabular}{|c|c|c|c|c|c|}
\hline \multicolumn{6}{|c|}{$\begin{array}{c}\text { Tabla } 1 \\
\begin{array}{c}\text { Distribuciones del vínculo en el grupo de madres drogodependientes y en el grupo de madres } \\
\text { elegido como grupo de control }\end{array}\end{array}$} \\
\hline & \multicolumn{5}{|c|}{ Categoría de Vínculo } \\
\hline & Ds & $\mathbf{F}$ & $\mathbf{E}$ & U & $N$ \\
\hline \multicolumn{6}{|c|}{ Grupo de madres } \\
\hline \multicolumn{6}{|l|}{ Dd } \\
\hline Frecuencias & 11 & 2 & 15 & & 28 \\
\hline Porcentajes & $39.3 \%$ & $7.1 \%$ & $53.6 \%$ & & 100 \\
\hline Frecuencias & 8 & 2 & 7 & 11 & 28 \\
\hline Porcentajes & $28.6 \%$ & $7.14 \%$ & $25 \%$ & $39.3 \%$ & 100 \\
\hline \multicolumn{6}{|c|}{ Grupo de control } \\
\hline Frecuencias & 9 & 23 & 8 & & 40 \\
\hline Porcentajes & $22.5 \%$ & $57.5 \%$ & $20 \%$ & & 100 \\
\hline Frecuencias & 9 & 21 & 5 & 5 & 40 \\
\hline Porcentajes & $22.5 \%$ & $52.5 \%$ & $12.5 \%$ & $12.5 \%$ & 100 \\
\hline
\end{tabular}

\footnotetext{
${ }^{4}$ El Modelo de Análisis Múltiple Log-lineal adoptado es un modelo Saturo que asume un Delta cercano a 0.5, sumado a las frecuencias observadas en cada una de las casillas de las tablas de contingencia con las que se calculan los valores estándar relativos a las estimaciones de los parámetros en el Modelo Log-Lineal. Para evitar que se acumulen los en el interior de cada una de las casillas de las tablas de contingencia se ha utilizado una corrección tipo Bonferroni del nivel estándar de Alfa de .0.5, según el procedimiento propuesto por Cristante (1992). La fórmula utilizada es, por tanto, .05/(r-1)(c-1), en la que ry c son respectivamente las rayas y las columnas de la tabla de contingencia analizada.
} 
culturales, dato que nos lleva a considerar el grupo de mujeres no drogodependientes elegido como un buen elemento de contraste para con la población clínica considerada (Simonelli, 1998). La diferencia entre las distribuciones de tres categorías de vínculo en los dos grupos es significativa desde el punto de vista estadístico (valor de Chi-cuadrado obtenido, $\chi_{-}[2, N=68]=$ 18.427, $p=0.01$ ]. Los resultados arrojados por el análisis Log-Lineal confirman todo lo que ha aparecido a nivel descriptivo, con un predominio de vínculo Complicado-Preocupado (E) y una proporción significativamente inferior de vínculo Seguro (F) en el grupo de las madres drogodependientes (Tabla 2). Los datos están, pues, en la misma línea en la que se sitúan los resultados del meta análisis de van IJzendoorn y BakermansKranenburg (1996) sobre grupos de madres pertenecientes a distintas poblaciones clínicas (siendo una de estas la de madres drogodependientes).

\begin{tabular}{|c|c|c|c|c|}
\hline \multicolumn{5}{|c|}{$\begin{array}{l}\text { Tabla 2. Análisis Log-Lineal. } \\
\begin{array}{c}\text { Valores estándar de Lambda para estimar la asociación entre las distribuciones } \\
\text { del grupo clínico y del grupo de control }\end{array}\end{array}$} \\
\hline & & \multicolumn{3}{|c|}{ Categoría de Vínculo } \\
\hline & Ds & $\mathbf{F}$ & $\mathbf{E}$ & $\mathbf{I}$ \\
\hline $\begin{array}{l}\text { Grupo de madres Dd } \\
\text { Grupo de control }\end{array}$ & $\begin{array}{r}1.783 \\
-1.783 \\
0.462\end{array}$ & $\begin{array}{c}-3.566^{* *} \\
3.566 * * \\
-1.077\end{array}$ & $\begin{array}{c}2.845^{* *} \\
-2.845^{* *} \\
0.936\end{array}$ & $\begin{array}{l}-1.666 \\
1.666\end{array}$ \\
\hline $\begin{array}{l}\alpha \text { (de la tabla })=.05 \\
\alpha \text { (de cada casilla) }=.025 \\
{ }^{*} \text { *alores Lambda Significativos }\end{array}$ & $\begin{array}{l}p<.05 \\
z \text { crit. }=1.96\end{array}$ & & & \\
\hline
\end{tabular}

Estas características también se evidencian en la distribución de cuatro categorías de vínculo (Tabla 1), que muestra una incidencia bastante elevada de la categoría No Resuelto en relación con un Duelo o un
Trauma (U) en el grupo clínico ( $n=11$ igual a 39,3\%; $\left.\left.\chi \_[3, N=68]=16.742, p=.001\right)\right)$, una proporción que difiere significativamente de lo que aparece en el grupo de control (Tabla 3).

\begin{tabular}{|c|c|c|c|c|c|}
\hline \multicolumn{6}{|c|}{$\begin{array}{l}\text { Tabla 3. Análisis Log-Lineal. } \\
\text { Valores estándar de Lambda para estimar la asociación entre las distribuciones } \\
\text { del grupo clínico y del grupo de control }\end{array}$} \\
\hline & & \multicolumn{4}{|c|}{ Categoría de Vínculo } \\
\hline & Ds & $\mathbf{F}$ & $\mathbf{E}$ & $\mathbf{U}$ & \\
\hline Grupo de madres Dd & 0.480 & $-3.348 * *$ & 1.319 & $2.324 * *$ & -1.129 \\
\hline Grupo de control & $\begin{array}{c}-0.480 \\
0.841\end{array}$ & $\begin{array}{l}3.348 * * \\
-0.260 \\
\end{array}$ & $\begin{array}{l}-1.319 \\
-0.665\end{array}$ & $\begin{array}{c}-2.324^{* *} \\
0.057\end{array}$ & 1.129 \\
\hline $\begin{array}{l}\alpha(\text { de la tabla })=.05 \\
\alpha \text { (de cada una de las casillas })=.017 \\
* * \text { Valores Lambda Significativos }\end{array}$ & $\begin{array}{l}p<.05 \\
z \text { crit. }=1.96\end{array}$ & & & & \\
\hline
\end{tabular}

Como se esperaba, en el grupo de madres drogodependientes la categoría de vínculo No Resuelto (U) aparece como característica distintiva respecto a la población general.

Un aspecto que debe subrayarse es el relacionado con el hecho de que, al parecer, las categorías de vínculo Complicado-Preocupado (E) y No Resuelto (U) que se manifiestan en el grupo de madres drogodependientes aparecen como factores descriptivos de sus dificultades para reelaborar los acontecimientos traumáticos que han caracterizado fuertemente sus historias vitales: ese dato es interesante porque indica que el trauma y sus consecuencias son, probablemente, los elementos más característicos de las experiencias personales y relacionales de las mujeres estudiadas, impulsándonos a investigar el papel que desempeñan no sólo en lo que concierne a la cualidad de su propio vínculo de vínculo, sino también en relación con la construcción de la relación con el niño durante los primeros años de vida.

De hecho la elevada proporción de vínculo inseguro en estos sujetos se puede explicar, en nuestra opinión, a través de diversos factores, destacando entre ellos la influencia precoz de ambientes de crecimiento poco adecuados. Lo cierto es que como también se deduce de los datos socio-demográficos, el contexto afectivo-relacional de las mujeres drogodependientes durante la infancia y la adolescencia se caracteriza por 
situaciones de sufrimiento grave presentes, sobre todo, en el interior de las familias de origen. En los estudios teóricos sobre el tema se apunta la hipótesis de que, al contrario de lo que sucede en situaciones de atención y de cariño, que protegen al sujeto y contribuyen a la construcción de la seguridad, los ambientes de crianza y de crecimiento poco sensibles, descuidados o marcados por el maltrato pueden constituirse, por el contrario, como factores de riesgo progresivo que pueden invalidar las experiencias de seguridad (Bowlby, 1988; Cicchetti \& Rizley, 1981). En términos de ecología del desarrollo (Bronfenbrenner, $1977,1979)$ se puede hablar de sistemas disfuncionales progenitor-hijo-ambiente que han provocado en las familias de origen de estas mujeres experiencias precoces de falta de atención y cuidado, siendo, al parecer, la carencia más significativa la de la protección que procede de las figuras de referencia primarias, que a veces es el punto de partida del peligro al que han estado expuestas.

Concretamente, el vínculo de tipo Complicado-Preocupado (E) se caracteriza por rasgos de pasividad, rabia y confusión que reflejan la dificultad del sujeto para integrar mentalmente los contenidos de la experiencia vivida con las figuras de vínculo. Esta categoría revela de hecho una incapacidad del sujeto adulto para moverse más allá de sí mismo con autonomía, sintiéndose confundido o, más bien, atravesado por relaciones precoces o experiencias negativas que siguen activas en la actualidad, operando a nivel de la organización mental (Main \& Goldwyn, 1994).

Estos aspectos se manifiestan de una manera especialmente evidente cuando se consideran cuatro categorías de vínculo en las que la no resolución se refiere más bien a experiencias traumáticas, y no tanto a duelos o pérdidas experimentadas durante la infancia. La fuerte presencia de la categoría No Resuelto (U) puede estar relacionada, de hecho, con los episodios de maltrato vistos o sufridos en el interior del núcleo familiar referidos por muchas de estas mujeres, episodios que se convierten en focos de sufrimiento y de pérdida durante los años de crecimiento. Estos episodios se caracterizan por actos y carencias que perturban al niño gravemente, constituyendo un atentado para su integridad corporal y para su desarrollo físico, intelectual y moral, y están relacionados con comportamientos negligentes y/o lesiones físicas, psíquicas o sexuales que, además, proceden de aquellas figuras de referencia que, por definición, deberían garantizar la integridad física y psicológica del niño, ocupándose de él, presentándose como una dramática contradicción de lo que deberían ser los cuidados, la nutrición y el amor que los padres prodigan a los hijos (Mrazek \& Mrazek, 1985).

Las experiencias de abuso vividas y experimentadas por el grupo de las mujeres drogodependientes se distinguen no sólo por su gravedad y por su dramatismo, sino sobre todo por el hecho de que se hayan repetido en el transcurso de la infancia y de la adolescencia, dato que parece haber determinado en ellas una incapacidad para prever y organizar comportamientos y estrategias defensivas al respecto. De hecho, en los casos en los que el progenitor se convierte en una fuente de terror para el niño, perdiendo la función protectora y de "base segura" (Ainsworth, 1973), a éste le resulta difícil construirse un cuadro integrado de estrategias cognitivas y emotivas porque no sabe qué ni cuándo esperar de él en los momentos en que quiere expresar su propio estado de necesidad. Los efectos de estos episodios siguen manifestándose en la vida actual de estas mujeres, inutilizando las modalidades cognitivas de procesamiento de la información procedentes del ambiente y de las relaciones, respondiendo a los abusos con comportamientos extremadamente desorientados y desorganizados.

Los maltratos y los abusos ponen también de relieve el cuerpo de la mujer como receptáculo de su falta de adaptación y de los traumas ligados a cómo se ha sentido cuidada por una parte y como instrumento con el que manifestar el sufrimiento psíquico por otra: las mujeres que hemos estudiado, que han sido golpeadas precoz y repetidamente en el cuerpo, parecen repetir ellas mismas los abusos de los que han sido víctimas, exponiéndose a condiciones de vida envilecedoras y de un desgaste tremendo para conseguir los medios necesarios para la adquisición de la droga. Nos referimos a las repetidas experiencias de prostitución y a los abortos voluntarios que caracterizan los años ligados a la drogodependencia. El cuerpo despreciado se convierte en un lugar de actuación, mientras que el cuerpo embarazado o el hijo recién nacido pasa a ser el lugar utópico del rescate, en un esfuerzo dramático por elaborar una identidad femenina que se quiere reencontrar y una condición de madre que se quiere averiguar a pesar de las experiencias pasadas durante el embarazo y en el periodo inmediatamente posterior al nacimiento del niño.

\section{EL VÍNCULO EN LOS HIJOS Y LA TRANSMISIÓN A TRAVÉS DE GENERACIONES SUCESIVAS}

Los datos que presentamos en la tabla 4 muestran un predominio de modelos de vínculo inseguro (Evitativo y Ambivalente) en los niños que han participado en la investigación. Sin embargo, estos resultados no deben interpretarse como una aparición de diferencias estadísticas respecto a los datos que arroja la población general italiana, que muestra una menor proporción de vínculo seguro y un incremento del modelo evitativo en relación con los datos que aporta la literatura internacional sobre el uso de la SSP (Fava Vizziello, 
calvo \& Simonelli, en prensa). De hecho, la comparación entre las distribuciones del grupo considerado y las de la investigación citada no ha evidenciado dife- rencias estadísticamente significativas, ni respecto a tres $\left(\chi \_[2, N=59]=4.457, p=.107, n s\right.$. $)$ ni respecto a cuatro categorías de vínculo $\left(\chi_{-}[3, N=59]=3.723, p=.292\right.$, ns. $)$.

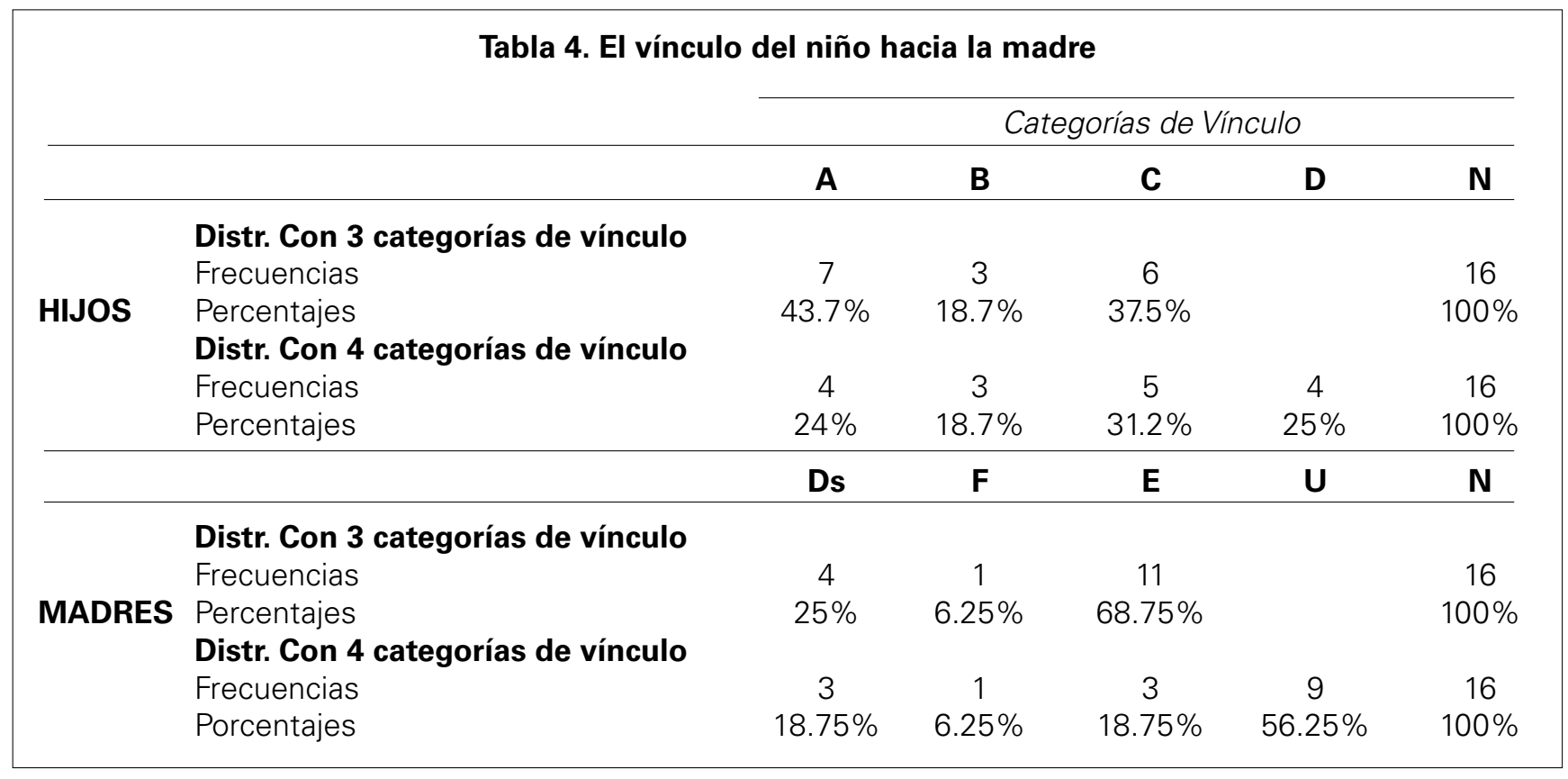

La valoración de los resultados obtenidos hasta aquí debe realizarse teniendo en cuenta dos advertencias: por una parte, la pequeñez del grupo que hemos estudiado, que limita la capacidad explicativa de los datos aportados por esta población de niños. Por otra, la tendencia hacia proporciones bastante bajas de vínculo seguro (B) que ya ha sido observada en grupos de sujetos italianos y europeos de la población general evaluados con la Stange Situation, es un fenómeno que merecería ser estudiado en el futuro con más profundidad, tanto a nivel intercultural como a nivel metodológico, para todo lo que concierne a la evaluación del vínculo del hijo hacia la madre durante el primer año de vida. En este sentido, el interés clínico del predominio de la inseguridad como estilo de vínculo predominante en este grupo específico de niños resulta especialmente interesante; además la homogeneidad de esta distribución frente a los resultados obtenidos en la población general italiana anima a una reflexión sobre el valor de adaptación de la cualidad del vínculo del niño en el ambiente de cuidados y de crecimiento en el que se ha insertado. En otras palabras, el valor de la seguridad y de la inseguridad tanto en grupos pertenecientes a la población general como a poblaciones clínicas necesitaría, según nuestra opinión, replantearse a la luz de factores contextuales, situacionales y afectivo-relacionales más amplios que contribuyan a aportar una visión evolutiva y clínica del estudio del vínculo que explique mejor la complejidad del tema de estudio al que nos estamos refiriendo. En este sentido, el concepto de adaptación interpsíquico e interpersonal debe considerarse un elemento clínico fundamental para la evaluación del bienestar relacional y afectivo de estos niños, desde el punto de vista del estudio del vínculo como estrategia elegida entre una vasta gama de modalidades relacionales entre la madre y el niño, gracias a las cuales puede adaptarse, por tanto, a las diversas condiciones ambientales (Hinde, 1982; Hinde \& Stevenson-Hinde, 1990). Para establecer el valor de protección o de riesgo de la cualidad del vínculo durante los primeros años de vida sería necesario tener en cuenta la capacidad de la díada para seleccionar la estrategia relacional más adecuada al contexto en el que se encuentra, dentro del repertorio de comportamientos de vínculo disponibles para ambos.

Al margen de las reducidas proporciones de seguridad que aparecen en este grupo, también es interesante resaltar que la distribución de los niños entre las dos categorías de vínculo inseguro (Evitativo y Ambivalente) parece equitativa, sin que se evidencie una tendencia dominante a la hora de elegir una estrategia determinada. Desde un punto de vista descriptivo, lo único que destaca es que en los niños la cantidad de vínculo Ambivalente $(\mathrm{C})$ es reducida $(37.5 \%)$ respecto a la elevada incidencia de este modelo en el grupo de las madres (68.7\%), un dato que parece introducir un elemento de variabilidad en la continuidad de los modelos de vínculo entre madre e hijo.

Igualmente, en lo que concierne a la distribución en cuatro categorías, la proporción entre vínculo Desorganizado/Desorientado (D) en los niños refuerza con bastante precisión los datos que aparecen en grupos de niños italianos de la población general (Ammaniti, et al., 1994; Fava Vizziello, et al., en prensa). Sin 
embargo, también en este caso la comparación cualitativa con las madres es lo que contribuye a proporcionar datos para reflexionar sobre la adaptación de los pequeños: la fuerte presencia de representaciones de vínculo No Resuelto (56.25\%) no parece, de hecho, un aspecto que se transmita linealmente al niño; es más, lo que se aprecia en esta tipología de vínculo es, más bien, cierta discontinuidad a través de las generaciones consideradas muy tranquilizadora en relación con la carga patógena del mundo interno de las madres sobre el crecimiento del niño.

En lo que respecta a la correspondencia entre el modelo de vínculo de la madre y el del niño, los datos cualitativos se han confirmado también a nivel estadístico: los resultados no han evidenciado asociaciones significativas para tres categorías de vínculo $(\chi[4, N=16]=3.108, p=.539)$. Por otra parte, el escaso número de sujetos considerados nos ha animado a repartir el grupo de las madres y de los hijos de acuerdo con la dicotomía Vínculo Seguro vs. Vínculo Inseguro (Tabla 5), que evidencia en cambio una tendencia a valores significativos $(\chi[N=16]=3.626, p=.05)$. El valor $K$ de Cohen obtenido es $K=.448, p=.032$, significativo pero más bien bajo. Este resultado subraya la escasa "fuerza" estadística de la asociación investigada, poniendo de relieve la casualidad que parece caracterizar la correspondencia entre la cualidad de las representaciones maternas y la del vínculo del niño.

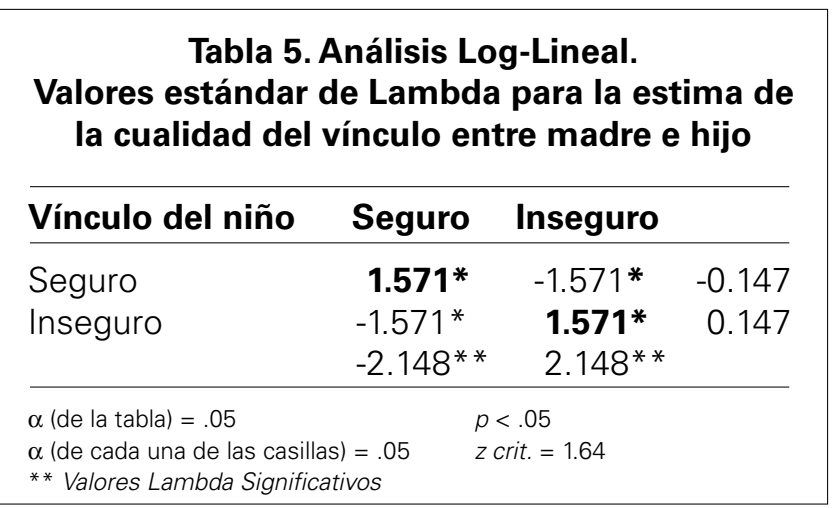

Los datos obtenidos tienen, en este sentido, una destacada relevancia clínica, aunque no tanto por lo que muestran sobre la transmisión del vínculo seguro o de los modelos inseguros en términos generales, ya que, de hecho, éstos no parecen suponer, en teoría, un riesgo evolutivo para el niño, además de que también caracterizan a grupos de niños criados en contextos adecuados. Lo que debe subrayarse es la interrupción de la continuidad de las modalidades de vínculo materno No Resueltas (U), porque éstas conllevan un potencial desorganizador para el niño en lo que respecta a su confianza en un ambiente estable desde el punto de vista afectivo.

En estas madres, los focos de afectos "asustados/asustantes" hacia ellas mismas y hacia el niño reenvían a experiencias reales vividas en el pasado y frecuentemente ligadas todavía al presente por sus efectos psicológicos y físicos a largo plazo. La transmisión de estos factores de desorganización al niño constituiría, de hecho, un factor de riesgo para su desarrollo, ya que su matriz relacional con el mundo representacional materno estaría contaminada por elementos luctuosos o traumáticos no elaborados o elaborables que se transmitirían al pequeño a través de comportamientos y cuidados poco adecuados.

En este sentido, el "gap" de transmisión identificado puede interpretarse como un aspecto de los niños que les protege de las vivencias internas de sus propias madres y de la posibilidad de que su condición no resuelta se establezca como núcleo relacional entre ambos.

\section{REFLEXIONES A MODO DE CONCLUSIÓN: UN LUGAR EN EL QUE CAMBIAR COMO FACTOR DE PROTECCIÓN.}

Los resultados del estudio permiten concluir que los niños observados muestran, en general, una adaptación, en términos de cualidades de vínculo a la madre, más favorable de lo que se esperaba, sobre todo si se tienen en cuenta las dificultades de reelaboración y organización de las representaciones internas de las vivencias personales que presentan sus madres. En este sentido, se puede establecer la hipótesis de que las comunidades en las que residen las parejas madre-hijo actúan como mediadoras en la transmisión lineal de la cualidad del vínculo y, también, en la transmisión de un factor más general de adaptación afectivo-relacional de cada uno de ellos y de la relación que existe entre los dos.

La explicación de todo esto se encuadra en lo que podríamos definir como la ampliación contextual de la teoría del vínculo, una ampliación que considera los lugares y los ambientes relacionales más extensos como verdaderos contextos, con una función de figuras de vínculo primarias. Desde este punto de vista, las comunidades se convierten, para las madres y para los hijos, en lugares de vínculo físico y psicológico en las que es posible estructurar relaciones afectivas nuevas y más adecuadas. Esta función puede desarrollarse gracias a la contención que aportan las propias comunidades, una contención que está relacionada con su rol de ambiente en el que se obtienen los cuidados más importantes, en el que se está protegido frente al peligro y en el que se encuentra una base segura para la exploración del ambiente.

Esta visión del vínculo permite por lo tanto considerar las comunidades, en su conjunto, como ambientes progenitores, y en ellas estas mujeres pueden sentir su maternidad como algo que recibe apoyo y 
sostén, en vez de como algo dificultoso que no son capaces de desarrollar y que, por lo tanto, sólo reporta castigo. Esto es posible por que las madres experimentan una importante regresión rol de hijas y de niñas necesitadas (de los trabajadores, de los psicólogos y de todos los que cuidan de ellas). El movimiento psíquico que consiente esta situación no está, ciertamente, desprovisto de ambivalencia, a causa del valor simbólico que supone el enfrentamiento con nuevas figuras progenitoras, que si bien esta vez están cerca y pendientes de las necesidades, también pueden ser severas y exigentes cuando es necesario. La posibilidad de experimentar sentimientos mezclados hacia estos nuevos "progenitores" institucionales puede permitir a estas mujeres recuperar los hilos relacionales y representacionales interrumpidos durante la infancia y la adolescencia, y permitirse a sí mismas una revisión de su propio pasado y de sus contenidos nocivos que resulta beneficiosa para la relación con el niño.

En el interior de la comunidad, por otra parte, las personas que se ocupan de los niños (educadores, psicólogos, neuropsiquiatras infantiles) desempeñan un rol que podríamos definir como de figuras de vínculo auxiliar, que pueden "diluir" y posponer los aspectos más conflictivos de la relación de los pequeños con sus madres.

Esa función crea un ambiente acogedor que está en condiciones de proteger el derecho de los niños a permanecer y crecer con sus madres aunque no sean perfectas, asumiendo que los vínculos primarios (aunque se hayan olvidado algunas veces en el transcurso de la vida) son, a pesar de todo, un patrimonio personal, irrenunciable e indeleble; paralelamente, el rol de los trabajadores se concreta también en la protección de los pequeños frente a los comportamientos poco adecuados de sus madres o, en el caso de que la intervención falle, teniéndose que acudir al desgarro de la adopción o de la separación definitiva del progenitor, frente a su incapacidad para cuidarles y para aceptar plenamente la maternidad.

Además, las comunidades asumen la condición de lugares en los que es posible mantener una constancia del objeto y de las relaciones, en las cuales es posible, por lo tanto, crecer en un contexto vital estable (que también significa dormir todas las noches en la misma cama, tener siempre los mismos juguetes y volver siempre a la misma casa después del jardín de infancia o de la escuela).

Todo esto permite a los niños acogidos experimentar un mundo en el que los adultos hacen lo que dicen o se esfuerzan por hacerlo, en el que las madres no trabajan siempre fuera de la ciudad o están en el hospital (como muchos abuelos o familiares al cuidado de los pequeños, que aprietan el paso para responder a las necesidades de éstos), en los que las madres están, son como son, pero están presentes.

\section{REFERENCIAS BIBLIOGRÁFICAS}

Ainsworth, M. D. S. 1973, The development of infant-mother attachment. In B. M. Caldwell \& H. N Ricciuti (Eds.) Review of Child Developmental Research. Chicago: University of Chicago Press, 3, 1094.

Ainsworth, M. D. S., Blehar, M. C., Waters, E., \& Wall, S. 1978, Patterns of Attachment. Hillsdale, NJ:Erlbaum.

Ammaniti, M., Candelori, C., Pola, M., Speranza, A. M., \& Tambelli, R. 1994, Influenze culturali e dinamiche relazionali nell'attaccamento infantile. Età Evolutiva, 47, 99109.

Azuma, C. D. \& Chasnoff, I. J. 1993, Outcome of children prenatally exposed to cocaine and other drugs: A path analysis of three-year data. Pediatrics, 92(3), 396-402.

Benoit, D. \& Parker, K. C. H. 1994, Stability and Transmission of Attachment across Three Generations. Child Devolopment, 65, 1444-1456.

Bowlby, J. 1988, Dalla teoria dell'attaccamento alla psicopatologia dello sviluppo. Rivista di Psichiatria, 23, 2, 5768.

Brofenbrenner, U. 1977, Toward an experimental ecology of human development. American Psychologist, 31, 513531.

Brofenbrenner, U. 1979, The ecology of human development: experiments by nature and design. Cambridge, MA: Harvard University Press.

Carlson, V., Cicchetti, D., Barnett, R., \& Braunwald, K. 1989, Disorganized/disoriented attachment relationship in maltreated infants. Developmental psychology, 25, 525-531.

Cavazzuti, G. B., Frigieri, G., \& Finelli, P. 1987, II follow-up del bambino nato da madre farmacodipendente. Bollettino per le Farmacodipendenze e I'Alcolismo, X, 6, 2028.

Cicchetti, D., \& Rizley, R. 1981, Developmental perspectives on the etiology, intergenerational transmission and sequelae of child maltreatment. New Directions for Children Maltreatment, 11, 31-55.

Cristante, F. 1992, Variabili qualitative in psicologia: metodi e modelli statistici. Padova: Upsel.

Crittenden, P. M. 1985, Maltreated infants: Vulnerability and resilience. Journal of Child Psychology and Psychiatry, 26, 85-96.

Fava Vizziello, G. M., Calvo, V., \& Simonelli, A. in press, Attachment patterns in early infancy and intercultural considerations: Study of an italian sample. Infant Mental Health Journal.

Finnegan, L. 1986, Neonatal abstinence syndrome: assessment and pharmacotherapy. In F. F. Rubatelli \& B. Granati (Eds.), Neonatal theraphy: an update. Elsevier Science Publistairs.

Fonagy, P., Steele, M., \& Steele, H. 1991, Maternal representation of attachment during pregnancy predict the organisation of infant-mother attachment at one year of age. Child Development, 62, 891-905. 
Fundaro, C. \& Salvataggio, E. 1987, Lo sviluppo del bambino di madre tossicodipendente. Bollettino per le Farmacodipendenze e I'Alcolismo, X, 6.

George, C., Kaplan, N., \& Main, M. 1985, Adult Attachment Interview. Manoscritto non pubblicato. Berkeley: University of California.

Hinde, R. A. 1982, Attachment: some conceptual and biological issues. In C. M. Parkers, \& J. Stevenson-Hinde (Eds.), The place of attachment in human behaviour. London: Tavistock.

Hinde, R. A., \& Stevenson-Hinde, J. 1990, Attachment: biological, cultural and individual desiderata. Human Development, 33, 1, 62-72.

Lester, B. M. \& Tronick, E. Z. 1994, The effects of prenatal cocaine exposure and child outcome. Infant Mentaal Health Journal, 15(2), 107-120.

Lutinger, B., Graham, K, Einarson, T. R., \& Karen, G. 1991, Relationship between gestational cocaine use and pregnancy outcomes: A meta-analysis. Teratology, 44, 405-414.

Main, M. \& Goldwyn, R. 1994, Adult Attachment Rating and classification systems. Manoscritto non pubblicato, Berkeley: University of California.

Main, M. \& Solomon, J. 1986, Discovery of a new, insecure-disorganized/disoriented attachment pattern. In T. B. Brazelton \& M. Yogman (Eds.), Affective development in infancy. Norwood: Ablex.

Main, M. \& Solomon, J. 1990, Procedures for identifying infants as disorganize/disoriente during the Ainsworth Strange Situation. In M. Greenberg, et al. (Eds.), Attachment in the preschool years: theory, research and intervention. Chicago: Univesity of Chicago Press.

Mayes, L. C., Granger, R. H., Bornstein, M. H., \& Zuckerman, B. 1992, The problem of prenatal cocaine exposure: A rush to judgment. Journal of the American Medical Association, 267, 406-408.

Mrazek, D. A. \& Mrazek, P. J. 1985, Child maltreatment. In M. Rutter \& H. Hersov (Eds.), Child and adolescent psychiatry: modern approaches (pp. 679-697). Oxford: Blackwell.

Oloffson, M. \& Buckley, W. 1983, Investigation of 85 children born by drug-addicted mothers. II Follow up 1-10 years after birth. Acta Pediatr. Scand., 72, 407-413.

Rodning, C., Beckwith, L., \& Howard, J. 1989, Characteristics of attachment organization and play organization in prenatally drug-exposed toddlers. Development and Psychopathology, 1, 277-289.

Sanderegger, T. \& Zimmermann, E. 1978, Adult behavior and adrenocortical function following neonatal morphine treatment in rats. Psychopharm., 56, 103-109.

Schneider-Rosen, K., Braunwald, K. G., Carlson, V., \& Cicchetti, D. 1985, Current perspectives in attachment theory: Illustration from the study of maltreated infants. In I. Bretherton \& E. Waters (Eds.), Growing points of attachment theory and research. Monographs of the society for research in child development, 50, (209), 194-210.

Simonelli, A. 1998, Adult Attachment Interview: nel ciclo della vita, lo studio dell'attaccamento. Tesi di dottorato, Università di Bologna.

Sroufe, L. A. \& Waters, E. 1977, Attachment as an organizational construct. Child Development, 54, 178-184.

van IJzendoorn, M. H. 1995, Adult Attachment Representation, Parental Responsiveness, and Infant Attachment: A Meta-Analysis on the Predictive Validity of the Adult Attachment Interview. Psychological Bulletin, 117, 3, 387-403.

van IJzendoorn, M. H. \& Bakermans-Kranenburg, M. J. 1996, Attachment representations in mother, father, adolescents, and clinical groups: A meta analytic search for normative data. Journal of Consulting and Clinical Psychology, 64, 1, 8-21.

van IJzendoorn, M. H. \& Bakermans-Kranenburg, M. J. 1997, Intergenerational transmission of attachment: A move to the contextual level. In L. Atkinson, \& K. J. Zuckerman (Eds.), Attachment and psychopathology (pp. 135-170). New York: Guilford Press.

van IJzendoorn, M. H., Goldberg, M., Kroonenberg, P. M., \& Frenkel, O. J. 1992, The relative effects of maternal and child problems on the quality of attachment: $A$ meta analysis of attachment in the clinical samples. Child Development, 63, 840-858.

Ward, M. J. \& Carlson, E. A. 1995, Association among adult attachment representations, maternal sensibility, and infant-mother attachment in a sample of adolescent mothers. Child Development, 66, 69-79.

Wilson, G. S., McCreary, R., Kean, J., \& Baxter, J. C. 1979, The developmental of preschool children of heroinaddicted mothers: A controlled study. Pediatrics, 63(1), 135-141.

Zacchello, F. \& Giaquinto, M. 1997, Figlio di madre tossicodipendente. Problemi clinici e assistenziali. In G.M. Fava Vizziello \& P. Stocco (Eds.), Tra genitori e figli la tossicodipendenza (pp.161-168). Milano: Masson.

Zeanah, C.H. 1992, L'esperienza soggettiva nella relazione di attaccamento: la prospettiva di ricerca. In M. Ammaniti \& D. N. Stern (Eds.), Attaccamento e psicoanalisi, Roma: Laterza.

Zuckerman, B. \& Bresnahan, K. 1991, Developmental and behavioral consequences of prenatal drug and alcohol exposure. Pediatric Clinics of North America, 38, 13871405.

Zuckerman, B., Frank, D. A., Hingson, R., Amaro, H., Levenson, S. M., Kayne, H., Parker, S., Vinci, R., Aboagye, K., Fried, L. E., Cabral, H., Timperi, R., \& Bauchner, H. 1989, Effects of maternal marijuana and cocaine use on fetal growth. New England Journal of Medicine, 320, 762-768. 